\title{
MORPHOLOGICAL CHARACTERISTICS OF ACELLULAR DERMAL MATRIX MANUFACTURING
}

DOI: 10.36740/WLek202103107

\author{
Larysa Ya. Fedoniuk', Ihor S. Kulyanda' ', Alina I. Dovgalyuk', Yuliia V. Lomakina'², Solomia B. Kramar', \\ Olena 0 . Kulianda ${ }^{1}$, Olesya 0. Valko $^{1}$ \\ 'HORBACHEVSKY TERNOPIL NATIONAL MEDICAL UNIVERSITY, TERNOPIL, UKRAINE \\ 2BUKOVINIAN STATE MEDICAL UNIVERSITY, CHERNIVTSI, UKRAINE
}

\begin{abstract}
The aim is to develop a method of the acellular dermal matrix manufacturing from pig's skin dermis while preserving the native structure. Materials and methods: Combination of physical and chemical effects on the dermis underlies in the process of an acellular dermal matrix manufacturing. Dermal collection of 1.0-1.3 mm thickness in pigs under 1 year of age from the back and partially from the lateral parts of the body was carried out. The 0.3-0.4 mm thickness layer of skin was previously removed from the relevant areas with help of a dermatome, which was physically and chemically treated. The maximum acellularization of the dermal matrix was achieved step-by-step and included four stages of skin processing: 1 - freeze-thaw process; 2 - glycerin dehydration; 3 - osmotic stress; 4 - cell residue removal by detergent. Results: Histological analysis of the of the pig's skin dermis revealed that after freeze-thaw cycles the collagen scaffold of the dermal matrix maintains its structural organization that was obtained as a result of the first stage of decellularization. On the second stage of decullalarization, the decreased number of fibroblastic cells was indicated. By means of this, the connective tissue elements that are represented by collagen fibers' multidirectional bundles retained their structural organization.

Fibroblasts lysis as basophilic stained elements was revealed in small amount of dermis on the third stage of the decellularization. Washing of lyophilized skin with nonionic detergent sodium dodecyl sulfate the complete absence of fibroblasts, epidermocytes in the hair follicles, endothelial cells in the wall of blood vessels was detected indicating the effectiveness of this reagent in removing residual products.

Conclusions: Suggested protocol for decullalarization of the pig's skin dermis is effective in removing nuclear and cellular structures from dermis. Particular protocols can be modified by increasing the temperature difference or changing the number of freeze-thaw cycles.
\end{abstract}

KEY WORDS: pig's skin, derma, acellular dermal matrix, morphology

Wiad Lek. 2021;74(3 p.l):418-422

\section{INTRODUCTION}

The influence of chemical compounds (about 5000), plants, physical factors, infectious agents, ectoparasites' bites and other insects, skin invasion by helminthes and other agents can lead to skin lesions [1,2]. Disease manifestations mostly depend on the irritant type, intensity and duration of its impact, repeated contacts, localization of the pathological process, physiological condition of tissues, general condition of the body and its protective and adaptive capabilities, whereas the curing depends on the depth and location of the lesion $[3,4,5]$.

Nowadays, more than 700 artificial wound dressings that are recommended for treatment of skin lesions are known, while the intensity of the new wound dressings development is not reduced. Wound dressings as well as drugs used in traditional treatment must be adapted to the stage of the wound process are currently available for clinicians [6].

Due to special mechanisms of influence, modern wound dressings actively affect the healing process. They are able to regulate the amount of exudate due to sorption, remove its excess by keeping the wound surface moist, provide gas exchange, maintain a certain temperature, change the $\mathrm{pH}$ of the burned surface, and prevent mechanical tissue injury [7].

Perspective way of various etiologies skin injuries repairment is the usage of decellularized (acellular) pig's skin. The main advantages of this biological material are the composition and structure of the pig' dermis that are as much as the patient's dermis [8]. It has been presented in various researchers that skin substitutes improve the quality of wound healing and functional outcomes. They are a useful tool in plastic and reconstructive surgery [9-13].

Currently, physical and chemical methods of decellularization are proposed that will allow the preserving of the structure and properties of xenografts $[14,15]$.

Acellular dermal matrix supports fibroblasts' infiltration, neovascularization, and epithelialization in the absence of a recipient immune response has been shown in conducted investigations [16].

It is also shown that cosmetic and functional results after usage of cutaneous xenografts for the treatment of skin defects significantly exceed the results after skin grafting with a perforated flap. Moreover, it promotes faster healing 


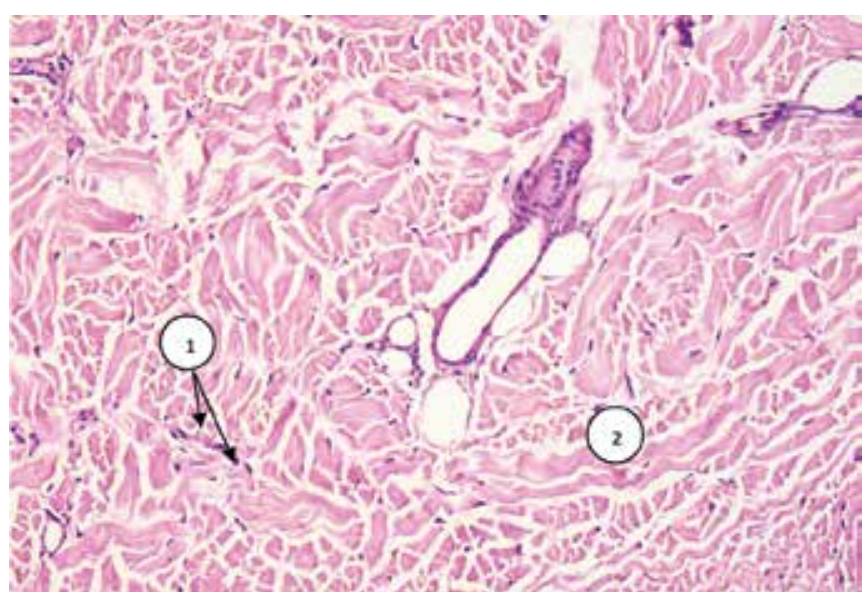

Fig. 1. Microscopic condition of lyophilized derma after freeze-thaw cycles. 1 - fibroblasts, 2 - collagen fibers. Hematoxylin and eosin. x200

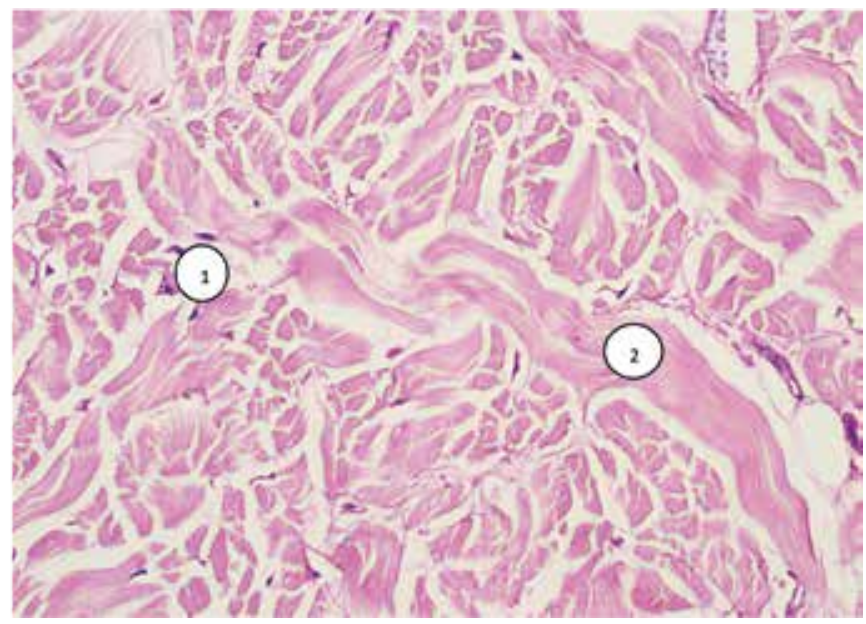

Fig. 3. Microscopic picture of the lyophilized derma after osmotic stress. 1 - residues of cellular material, 2 - collagen fibers. Hematoxylin and eosin. X100

of donor wounds, as it requires thinner autodermal grafts $[17,18]$. However, decellularization methods usually have opposite effects: extremely aggressive removal of immunogenic components can destroy the structure and composition of the tissue, while more gentle techniques can preserve the immunogenicity of the tissue.

Particularly, methods of obtaining skin substitutes do not have a common carrier of cellular structures, which would possess: biocompatibility, would create an optimal microenvironment for wound regeneration, would have the absorption capacity for wound exudate, would prevent entering and developing of microorganisms, would be permeable to water vapor and air, wouldn't dry the bottom of the wound, would be elastic, would simulate a surface with a complex texture.

\section{THE AIM}

To create a method of acellular dermal matrix (ADM) manufacturing from dermis of pig skin with preservation of native structure.

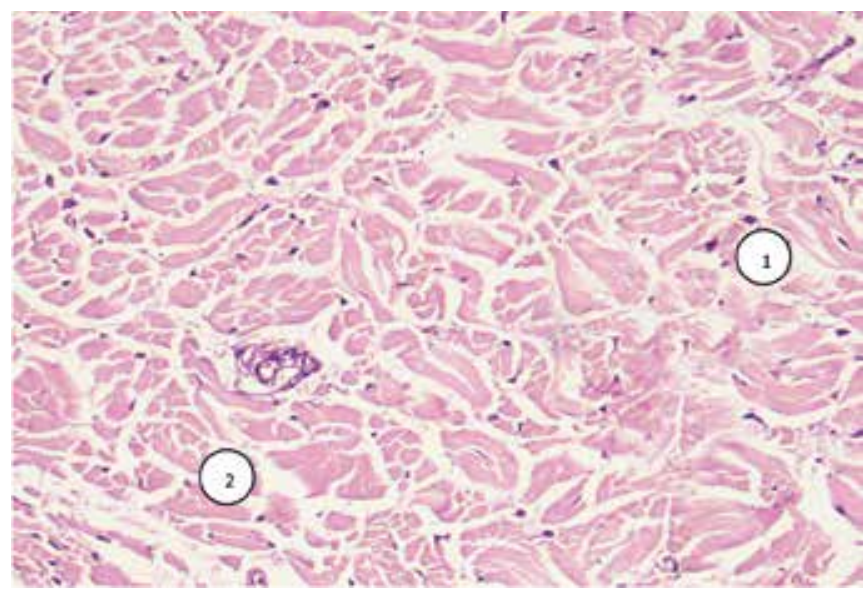

Fig. 2. Histological picture of the lyophilized derma after glycerol dehydration. 1 - damaged fibroblasts, 2 - collagen fibers. Hematoxylin and eosin. X200

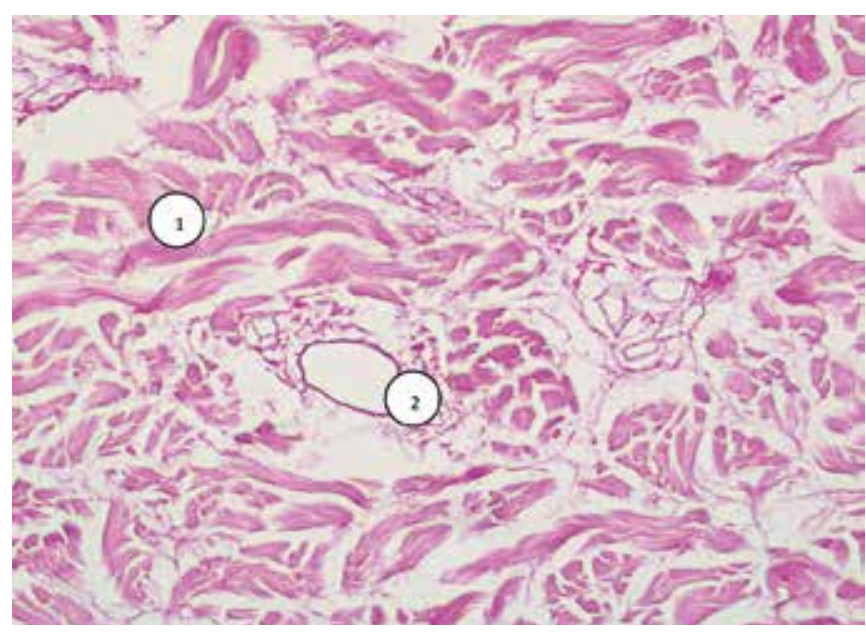

Fig. 4. Histological condition of reticular layer of lyophilized derma after rinsing with sodium dodecyl sulfate (SDS). 1 - collagen scaffold, 2 - vessels wall. Hematoxylin and eosin. X100

\section{MATERIALS AND METHODS}

The process of ADM manufacturing has been a combination of physical and chemical effects on the dermis. In pigs under 1-year of age 1.0-1.3 mm thickness derma was collected from the back and partially from the lateral parts of the body. Previously with help of dermatome the particular 0,3-0,4 mm thickness skin layer was removed and was proceeded by physical and chemical influence. Step by step the achieving the maximum acellularity of the dermal matrix was carried out and included 4 stages of skin treatment: 1 - the process of freezing-thawing; 2- dehydration with glycerin; 3 - osmotic stress; 4 - washing with detergent of the remaining cells. Freezing was conducted in liquid nitrogen $\left(-196^{\circ} \mathrm{C}\right)$, thawing in $37^{\circ} \mathrm{C}$ water. This cycle was repeated three times. Subsequently, three times, the skin was covered with a solution of glycerin, osmotic stress was performed by alternating immersion of the dermis in distilled water and hypertonic solution. The skin was then treated with $1 \mathrm{M} \mathrm{NaOH}$ solution.

On the final stage, residues of cell breakdown products, proteins and other components of the extracellular matrix were removed by $1 \%$ sodium dodecyl sulfate (SDS).

Afterwards, the skin underwent freeze-drying. 
To evaluate the effectiveness of the decellularization protocol, morphological analysis of the dermis was performed using light microscopy. For this purpose, samples of native $(n=6)$ and decellularized $(n=6)$ dermis were fixed in $10 \%$ neutral formalin, filled with paraffin. The obtained $5 \mu \mathrm{m}$ samples were dewaxed and stained with hematoxylin and eosin (H\&E; Sigma-Aldrich, Inc., St Louis, MO, USA) for detection of nuclear, cellular and extracellular materials.

\section{RESULTS}

According to the results of light optical microscopy in the lyophilized pigs' dermis before the beginning of decellularization, any signs of autolytic and necrobiotic changes, as well as morphological disorders of its structural organization were observed.

It was revealed under microscopic examination of lyophilized skin that the dermis and hypodermis are located under the multilayered squamous corneal epithelium (epidermis) lying on the basement membrane. The papillary and the reticular layers of the dermis are represented by connective tissue. In the bulk of the cells that form these layers, well-defined nuclei and cytoplasm of fibroblasts, as well as clearly visualized nuclei of epidermocytes in the hair follicle.

In overwhelmingly of histological specimens, the collagen fibers of the dermis are contoured, form a network and are differently localized in the papillary layer of the dermis.

The deeper dermis parts (reticular layer) represented with an insignificant swelling and homogenization of collagen fibers' particular sections, and in some cases - their fragmentation. The connective tissue framework is also depicted by clearly contoured thin elastic fibers.

The reticular and papillary layers of the dermis are formed by fibrous connective tissue, where collagen fibers are in dominance was revealed in the dermis of pig skin after freeze-thaw cycles. Thick bundles that are located in different directions in the intercellular matrix and have an oxyphilic color represent the fibrous component of the reticular layer (Fig. 1).

The cellular composition is illustrated mainly by fibroblasts, the nuclei of which have a basophilic color after hematoxylin and eosin staining.

It is shown that the collagen scaffold of the dermal matrix obtained as a result of the first stage of decellularization retains its structural organization.

A decreased number of fibroblastic cells was revealed on the second stage of decellularization - dehydration with polyhydric alcohol glycerol (Fig. 2). In this case, the connective tissue elements of the dermis, which are represented by multidirectional bundles of collagen fibers in the papillary and reticular layers, retain their structural organization, which is confirmed by light microscopy.

The second stage of decellularization - dehydration with polyhydric alcohol glycerol - revealed decreased number of fibroblastic cells.

The second stage of decellularization - dehydration with polyhydric alcohol glycerol - found a decrease in the number of fibroblastic cells (Fig. 2).
In this case, the connective tissue elements of the dermis, which are represented by multidirectional bundles of collagen fibers in the papillary and reticular layers, retain their structural organization, which is confirmed by light microscopy.

Meanwhile, light microscopy confirmed that the connective tissue elements of the dermis represented by multidirectional bundles of collagen fibers in the papillary and reticular layers, maintain their structural organization.

As a result of the third stage of decellularization (osmotic stress) lysis of fibroblastic cells under microscopic examination of histological specimens was revealed. This can be explained by the fact that hypotonic solutions easily cause the dissolution of cellular material due to simple osmotic effects with minimal changes in the molecules of the matrix and its architecture [19], hypertonic saline dissociates DNA from proteins [20]. The data obtained by light microscopy revealed a small number of basophilically stained elements in the dermis, which can be regarded as residues of structural components of the cells (Fig. 3).

The final stage of the study involved rinsing the lyophilized skin with a nonionic detergent sodium dodecyl sulfate (SDS). This contributed to the removal of residues of cell decay products, which indicates the effectiveness of this reagent.

Light microscopy data show the complete absence of fibroblastic cells, epidermocytes in the hair follicles, endothelial cells and smooth myocytes in the wall of blood vessels (Fig. 4).

\section{DISCUSSION}

It is known that freeze-thaw cycles do not affect the content of collagen and glucosaminoglycans (GAG), as well as the mechanical strength of biological material [21]. However, $88 \%$ of DNA in cells remains unchanged after such physical exposure. Thus, this physical effect on lyophilized skin can be considered as a trigger for damaging effects on the cellular structures of the dermis.

At the stage of decellularization of the dermis, alcohols, in particular glycerin, contribute to the structural reorganization of cells by dehydration and lysis [22,23]. Alcohols will also dissolve phospholipids, which are a part of cell membranes. However, attention should be paid to the negative effects of alcohols on tissues due to their ability to precipitate proteins, as well as ultrastructural extracellular matrix damage they can cause $[24,25,26]$.

To obtain the maximum osmotic effect (third stage of decellularization), the tissues should alternatively be immersed in hyper- and hypotonic solutions for several cycles, which helps to leach the cells' residues from the tissue after lysis.

The step of the lyophilized skin washing with a nonionic sodium dodecyl sulfate (SDS) detergent proved to be effective for the complete removal of cellular material from the dermis, as this led to the solubilization of cell membranes and their complete leaching from the tissues. Increasing the exposure and concentration in the decellularization pro- 
tocol by SDS also leads to complete removal of cell nuclei [27]. Removing of extracellular matrix proteins and DNA by usage of detergents increases with exposure time and varies depending on the organ subunit, tissue type and donor age [28]. SDS is generally more effective for elimination of cell residues from tissue than other detergents, although is also more destructive for the extracellular matrix $[29,30]$. Therefore, the SDS concentration and exposure for each tissue should be carefully and accurately fitted.

Removal of cellular material from the dermis of pig skin as a potential coating for the wound surface significantly reduces the antigenic properties of the material, ensuring its constant engraftment to the wound area with vascular germination and gradual filling of the recipient's own cells. Preservation of the structure of the collagen matrix promotes angiogenesis and cell migration [31, 32]. In the future, decellularized dermal matrices are considered as one of the main scaffold options for creating a bioengineered autologous skin equivalent.

\section{CONCLUSIONS}

Proposed protocol of pig' skin derma decellularization is effective for nuclear and cellular particles elimination from the cell, as it has been concluded in this research. Particular protocols could be changed by increasing the temperature differences or changing the number of freeze-thaw cycles.

\section{REFERENCES}

1. Bojchuk T.M., Yermolenko S.B., Fedonyuk L.Ya., et al. The magnitude of linear dichroism of biological tissues as a result of cancer changes. Proc. SPIE. Materials of the 10th International Conference Correlation Optics. 2011;8338,ID8338-54:83381 K1-K7.

2. Vaǐnshteľn S.G., Masik A.M., Zhulkevich I.V. Food fiber-research results and outlook. Vopr Pitan. 1988;6:8-12.

3. Xu S.J. et al. Differences of wound contraction and apoptosis in fullthickness burn wounds repaired with different artificial dermal stent in pigs. Chinese journal of reparative and reconstructive surgery. 2010;48(11):856-860.

4. Bosco F. et al. The use of banked skin in the Burns Centre of Verona. Blood Transfus. 2011;9(2):156-161. D0I: 10.2450/2011.0107-09.

5. Vănshteǐn S.G., Zhulkevich I.V., Petropavlovskiĭ G.A., Kotelnikova N.E. Protective properties of microcrystalline cellulose in experimental diabetes mellitus in rats. Biull Eksp Biol Med. 1987;103;2:167-168.

6. Fedonyuk L.Ya., Oleshchuk A.M., Sas L.M., et al. Polypragmasy: from paediatrics to geriatrics. Clinical Practice in Pediatrics. 2018;1(13):77-82.

7. Percival S.L. et al. The effects of $\mathrm{pH}$ on wound healing, biofilms, and antimicrobial efficacy. Wound Repair and Regeneration. 2014;22(2):174-186.

8. Debeer S., Le Luduec J.B., Kaiserlian D. et al. Comparative histology and immunohistochemistry of porcine versus human skin. Eur. J Dermatol. 2013;23(4):456-466.

9. Chua A.W., Khoo Y.C., Tan B.K. et al. Skin tissue engineering advances in severe burns: review and therapeutic applications. Burns Trauma. 2016;4:3.

10. Tracy L.E., Minasian R.A., Caterson E.J. Extracellular matrix and dermal fibroblast function in the healing wound. Adv. Wound Care (New Rochelle). 2016;5:119-136.
11. Chaudhari A.A., Vig K., Baganizi D.R. et al. Future prospects for scaffolding methods and biomaterials in skin tissue engineering: a review. Int J Mol Sci. 2016;17:1974.

12. Sheikholeslam M., Wright M.E.E., Jeschke M.G. et al. Biomaterials for skin substitutes Adv. Healthc Mater. 2018;7:1700897.

13. Cui H., Chai Y., Yu Y. Progress in developing decellularized bioscaffolds for enhancing skin construction. J Biomed Mater Res A. 2019;107(8):1849-1859.

14. Hrebikova H., Diaz D., Mokry J. Chemical decellularization: a promising approach for preparation of extracellular matrix. Biomed Pap Med Fac Univ Palacky Olomouc Czech Repub. 2015;159(1):12-7.

15. Crapo P.M., Gilbert T.W., Badylak S.F. An overview of tissue and whole organ decellularization processes. Biomaterials. 2011;32 (12):3233-3243.

16. Riau A.K., Beuerman R.W, Lim L.S. et al. Preservation, sterilization and de-epithelialization of human amniotic membrane for use in ocular surface reconstruction. Biomaterials. 2010;31(2):216-225.

17. Olczyk P., Mencner L., Komosinska-Vassev K. The role of the extracellular matrix components in cutaneous wound healing. BioMed Res Int. 2014; 2014:747584.

18. Volk S.W., Iqbal S.A., Bayat A. Interactions of the extracellular matrix and progenitor cells in cutaneous wound healing. Adv Would Care. 2013;2(6):261-272.

19. Xu C.C., Chan R.W., Tirunagari N.A. Biodegradable, acellular xenogeneic scaffold for regeneration of the vocal fold lamina propria. Tissue Eng. 2007;13(3):551-566.

20. Cox B., Emili A. Tissue subcellular fractionation and protein extraction for use in mass-spectrometry-based proteomics. Nat Protoc. 2006;1(4):1872-1878.

21. Xing Q., Yates K., Tahtinen M. et al. Decellularization of fibroblast cell sheets for natural extracellular matrix scaffold preparation. Tissue Engineering-Part C: Methods. 2015;21(1):77-87.

22. Prasertsung I., Kanokpanont S., Bunaprasert T. et al. Development of acellular dermis from porcine skin using periodic pressurized technique. J Biomed Mater Res B Appl Biomater. 2008;85(1):210-219.

23. Alekseyenko N. S. , Andriychuk V. M., Fedoniuk L. Ya., et al. Peculiarities of trunk skin and fat flexures changes of rural and city youth in the conditions of the educational process. Wiad. Lek. 2020;9(73):2017-2020.

24. Levy R.J., Vyavahare N., Ogle M. et al. Inhibition of cusp and aortic wall calcification in ethanol- and aluminum-treated bioprosthetic heart valves in sheep: background, mechanisms, and synergism.J HeartValve Dis. 2003;12(2):209-216.

25. Gorschewsky 0., Klakow A., Riechert K. et al. Clinical comparison of the Tutoplast allograft and autologous patellar tendon (bone-patellar tendon-bone) for the reconstruction of the anterior cruciate ligament: 2- and 6-year results. Am J Sports Med. 2005;33(8):1202-1209.

26. Gorschewsky 0., Puetz A., Riechert K. et al. Quantitative analysis of biochemical characteristics of bone-patellar tendon-bone allografts. Biomed Mater Eng. 2005;15(6):403-411.

27. Alekseyenko N.S., Andriychuk V.M., Radoha R.V., et al. Comparative characteristics of the parameters' changes of skin and fat flexures thickness of extremeties at youth under the condition of higher education. Wiad. Lek. 2020;10(73):2214-2218.

28. Nakayama K.H., Batchelder C.A., Lee C.I., Tarantal A.F. Decellularized rhesus monkey kidney as a three-dimensional scaffold for renal tissue engineering. Tissue Eng Part A. 2010;16(7):2207-2216.

29. Yang B., Zhang Y., Zhou L. et al. Development of a porcine bladder acellular matrix with well-preserved extracellular bioactive factors for tissue engineering. Tissue Eng Part C Methods. 2010;16(5):1201-1211. 
30. Du L., Wu X., Pang K., Yang Y. Histological evaluation and biomechanical characterisation of an acellular porcine cornea scaffold. Br J Ophthalmol. 2011;95(3):410-4.

31. Funamoto S., Nam K., Kimura T. et al. The use of high-hydrostatic pressure treatment to decellularize blood vessels. Biomaterials. 2010;31(13):3590-3595.

32. Riau A.K., Beuerman R.W., Lim L.S. et al. Preservation, sterilization and de-epithelialization of human amniotic membrane for use in ocular surface reconstruction. Biomaterials. 2010;31(2):216-225.

The research was performed as part of the initiative research work "Study of the regenerative potential of mesenchymal stem cells in tissue damage in the experiment and the prospects for their therapeutic use", the state registration number: $0120 U 104146$.

\section{ORCID and contributionship:}

Larysa Ya. Fedoniuk: 0000-0003-4910-6888 A, D, F

Ihor S. Kulyanda: 0000-0003-2537-9136 ${ }^{B, E}$

Alina I. Dovgalyuk: 0000-0003-3976-0245 ${ }^{A, D}$

Yuliia V. Lomakina: 0000-0002-8020-5254 ${ }^{D, F}$

Solomia B. Kramar: 0000-0003-3654-4950 E, F

Olena O. Kulianda: 0000-0001-6197-9046 ${ }^{B, C}$

Olesya O. Valko: 0000-0001-8648-6571 C, E

\section{Conflict of interest:}

The Authors declare no conflict of interest.

\section{CORRESPONDING AUTHOR}

Larysa Ya. Fedoniuk

I. Horbachevsky Ternopil National Medical University

Valova street 9, 46000, Ternopil, Ukraine

tel: +380673999143

e-mail: Fedonyuk22Larisa@gmail.com

Received: 12.10 .2020

Accepted: 23.02.2021

A - Work concept and design, B - Data collection and analysis, C - Responsibility for statistical analysis,

D-Writing the article, $\mathbf{E}$-Critical review, $\mathbf{F}$ - Final approval of the article 